\title{
GPS 控制测绘技术在地理信息系统中的应用
}

\author{
郡芳萍 王佳琦 \\ 湖州吴兴东成测绘有限公司 \\ DOI:10.32629/gmsm.v3i1.564
}

[摘要] 伴随着科学技术发展而产生的,是新生代的科学技术产品。GPS高新卫星定位科技,给我国的科学技术发展带来了新一代的发展浪潮。 GPS是一种利用卫星进行准确定位的高科技测绘技术, 它既可以对空间上进行准确定位, 又可以对地面进行准确定位。涵盖面及广,已经完全取 代了传统落后的只定位一个方向的定位仪器。通过GPS控制测绘技术的应用,对于我国的地理信息系统是一次重大性的技术革新。

[关键词] 地理信息系统; GPS; 控制测绘技术

\section{GPS 系统控制测绘技术的应用特点}

\section{1 高效性}

与传统定位器相比较, 该系统控制测绘技术工作效率高, 可以对某个 位置坐标进行一次性获取, 这其中也包括三维坐标, 并强化其定位效果。但 在传统测量操作之中, 需要利用两种或两种以上的测量方式, 才能将上述 测量工作完成。具体测量区域网中, GPS要比传统方式取点更随意, 对于不 符合要求的, 还能进行有效的补点操作。随着新技术的不断应用, 工程建设 效率能够得到显著性提升, 在节省时间的同时, 还能避免相关人力和财力 的浪费。

\section{2 实时性}

在实际工作过程中, 客户很可能会临时更改需求, GPS控制测绘技术在 该方面具备明显优势。相关工作人员应按照实际变化情况, 将需求的实时 性特点表现出来, 实现对设计方案的全面修改, 在满足客户要求的同时, 降 低后期数据更改而产生的损失, 强化其工作效率。

\section{GPS 控制测绘技术在地理信息系统中的应用}

2.1 导航系统与地理系统的融合

测绘数据结果的比对十分重要, 在确保数据库整合的同时, 对组织结 构和组织要素等进行有效分类, 最终实现信息内容的高效集中。除此之外, 在实际GPS和GIS作用下, 能够通过二维、三维技术, 将实际降雨的情况呈现, 绘制成相应图形。例如, 在部分城市中的湿地公园附近, 往往会建设很多工 厂, 从而导致大量废物被排放到河水之中, 加剧了周围环境的污染程度。在 此过程中, 相关工作人员需要根据实际GIS数据特征, 将具体工厂污染的扩 散图模拟出来, 工作人员可以依托管道、速度等数据, 使模拟画面更加完整, 增强其精度。在GPS应用之中, 由于自身具备较强的空间定位功能, 可以确 保该项技术能够与地理信息系统实现融合, 并对采集到的精准数据结果进 行空间描述综合分析, 进而将现代数字管理特点呈现出来。随着城市的不 断发展, 信息技术的作用越来越明显, 需要展示出数据的精准性, 而GPS和 GIS的结合可以满足上述要求。

2.2 GPS导航在GIS地理信息系统中的应用

在现代生活和社会发展过程中, 手机成为人们生活中不可缺少的重要 组成部分, 尤其是在车载地图模拟应用上, 能够帮助人们对信号信息进行 直接接收, 将信息功能展示出来, 并将其与移动定位画面有效结合, 将信息 在屏幕上显示出来, 将后续遥感数字图像和地理数字地图等功能结合。一 般来说, 对于电子地图的制作, GPS系统支持显得尤为重要, 在实际信息获 取上, 最为常见的形式包括以下几方面, 即遥感获取、子化获取以及GPS导 航获取等, 为后续操作创造更多有利条件。

\subsection{RTK定位技术}

可借助于快速动态和快速静态, 实现连续性定位操作, 并确保测站 点的三维定位功能, 确保空间定位能够精准到CoM, 甚至更高。另外, 在实 际GPS控制测量数据上, 主要以传输流动站为主, 进而实现全面的数据连 接操作, 实现对基准站数据的全面接收和管理。在实际RTK控制测绘技术 作用下, 可以将观测值和基准站坐标信息与GPS一起传送到流动站之中, 最终实现GPS数据和RTK数据资料的有效存储和分析, 为后续工作开展创 造有利条件。

2. 4 实际生活中的应用

GPS技术在实际生活中发挥了很大作用, 如GPS导航在应用时, 能够为 人们的日常出行提供路线规划, 并将实际位置准确发送到用户手中。与此 同时, 在整个出行过程中, 相关工作人员还需要将所经过的地点、时间等方 式信息全部传达给用户, 强化出行的安全性和可靠性。整个GPS控制测绘技 术应用, 还能被应用到财产监控操作之中。现阶段, 很多智能手机均具备相 应的定位功能, 只要能够与智能终端软件相结合, 人们便能够利用GPS来获 取手机位置, 将信息来源直接锁定, 该项应用在打击犯罪上得到了充分应 用, 可以帮助警察提升办案效率。除此之外, 工作人员还能将GPS控制测绘 技术和地理信息系统整合在一起, 构建出一套完整的城市电子地图。

2.5 定界勘测中的应用

工作人员可以将GPS控制测绘技术与城镇地籍测量工作相结合, 协助 城镇建设的有效开发和利用, 并以此为定界手段, 为后续提供地理参考数 据。站在实际地籍勘测角度来说, 经常会遇到复杂地形, 勘测面积也较大, 此时, 在GPS技术的帮助下, 可以降低测量过程的难度, 同时还能维护地籍 定界的准确性。

\section{3 结语}

随着科技不断进步, 各行业对GPS测绘技术的要求也越来越高, 以目前 发展情况来看, 快速的收集数据信息、准确的测量结果、实时随意的定, 数字化测绘方式将取代传统测绘方法, 不断改进和弥补测量缺陷, 向着多 元化、现代化方向发展。

\section{[参考文献]}

[1]韩丽荣.地理信息系统中 GPS 控制测绘技术的应用 [J]. 时代农 机,2015,42(11):12-13.

[2]王留洋.地理信息系统中GPS控制测量技术的价值研究 [J].江西建 材,2016,(10):235.

[3]苑占清.GPS控制测绘技术在地理信息系统中的应用 [J].海峡科技 与产业,2016,(02):59-60. 